\title{
Using Chandra/LETG Spectra to Probe Stellar Coronae
}

\author{
Gaitee A. J. Hussain \\ ESTEC, ESA, P.O.Box 299, 2200 AG, Noordwijk, The Netherlands
}

Nancy Brickhouse, A. K. Dupree, Adriaan A. van Ballegooijen

Harvard Smithsonian CfA, 60 Garden St, Cambridge MA02138, USA

Andrew Collier Cameron, Moira Jardine

School of Physics \& Astronomy, Univ. of St Andrews, Fife, UK

Jean-Francois Donati

Laboratoire d'Astrophysique, Observ. Midi-Pyrénées, Toulouse, France

\begin{abstract}
.
We probe the relationship between surface magnetic fields and the $\mathrm{X}$-ray emitting corona in the rapidly rotating star, AB Dor. Circularly polarised spectra have been inverted to produce a surface (photospheric) magnetic field map. This surface map has been extrapolated to model AB Dor's coronal field topology and X-ray light curve. Chandra/LETG light curves of $\mathrm{AB}$ Dor from the same epoch show intrinsic variability at the $30 \%$ level. Period analysis indicates a fraction of this is due to rotational modulation. We measure velocity shifts in emission line centroids as a function of $\mathrm{P}_{\text {rot }}$ and find evidence of rotational modulation (max. vel. $\sim$ $40 \pm 13 \mathrm{~km} \mathrm{~s}^{-1}$ ). This modulation may indicate the presence of a localised $\mathrm{X}$-ray emitting region at mid-to-high latitudes.
\end{abstract}

\section{Introduction}

We present the first results from a co-ordinated optical and X-ray campaign to probe the corona of a rapidly rotating, active cool star. X-ray and EUV observations from the Chandra, XMM and EUVE satellites reveal a picture of stellar coronae unlike anything on the Sun. Emission measures of about $10^{52} \mathrm{~cm}^{-3}$ are found in plasma at temperatures of $8 \mathrm{MK}$ and beyond, even in relatively quiescent coronae (e.g. Sanz-Forcada et al. 2003). Emission measures peak at $10^{50} \mathrm{~cm}^{-3}$ at $2.5 \mathrm{MK}$ on the Sun in active regions. (Drake et al. 2000). While the thermal properties of these active stellar coronae are increasingly well-determined, we have yet to establish where this emission originates and to confront observations with theoretical models.

Previous studies provide evidence for both extended and compact coronae in active cool stars. The extended coronal model is supported by observations 

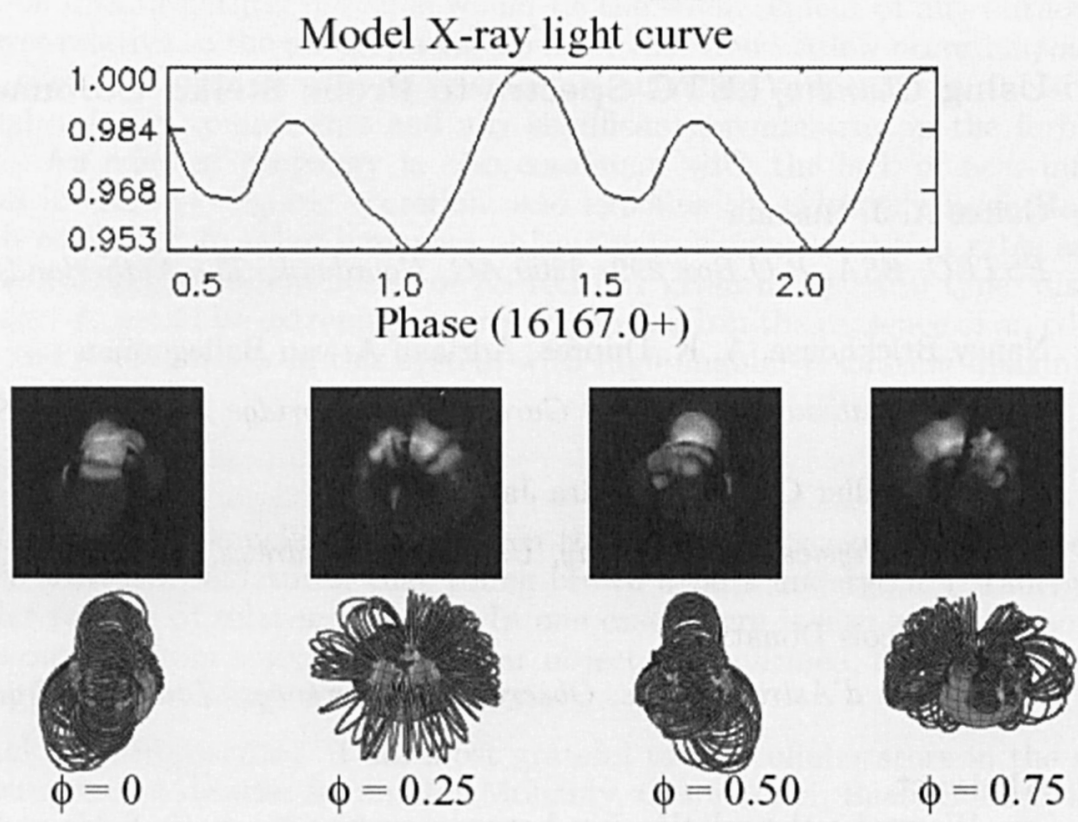

Figure 1. [bottom row] Surface magnetic field maps from 2002 Dec 11 are extrapolated to model $A B$ Dor's 3-D coronal field, generate " $X$-ray snapshots" [middle row], and predict its $X$-ray light curve [top row].

of fast moving $\mathrm{H} \alpha$ absorption transients in young stars. These are caused by cool prominences rigidly corotating with the star at heights near and beyond the Keplerian corotation radius (Collier Cameron et al. 2001). Further support for extended coronae comes from time-resolved HST spectroscopy of the eclipsing binary, V471 Tau (K2V+DA). The hot white dwarf acts as a probe of circumstellar material around the K2 star enabling Walter (this volume) to detect hot plasma $\left(10^{4}<T<10^{5} \mathrm{~K}\right)$ at heights greater than $2 R_{*}$.

Support for compact coronae comes from X-ray light curves of the eclipsing binary, Algol (B8V+K2III). Flaring regions are found to have heights, $0.1<\mathrm{H}<0.6 \mathrm{R}_{*}$ above the $\mathrm{K}$ component (Schmitt \& Favata 1999, Schmitt et al. 2003). Maggio et al. (2000) find evidence for a small flaring region $\left(H<0.3 \mathrm{R}_{*}\right)$ on the single, K0V, AB Dor. likely to be located near its pole. Electron density diagnostics measured in $10^{7} \mathrm{~K}$ plasma in active stars are typically very high $\left(n_{e}>10^{12} \mathrm{~cm}^{-3}\right)$. Densities this high are more easily explained in terms of compact X-ray emitting regions (e.g. Sanz-Forcada et al. 2003).

Brickhouse et al. (2001) find evidence for compact, quiescent coronae in active stars in a Chandra/HETG study of the contact binary, 44i Boo (G0V \& G0V), spanning over $2.5 \mathrm{P}_{\text {rot }}$. Flaring emission is filtered out and velocity shifts in line centroids of emission line profiles are measured as a function of rotation phase. Rotational modulation in the quiescent corona is detected, indicating a compact emitting area near the pole of the primary. We particularly want to follow up 
on this study in order to learn whether the picture of compact stellar coronae is applicable to other active cool stars or whether it is exclusive to contact binaries.

\subsection{AB Doradus}

AB Doradus (K0V, $m_{V}=6.9, \mathrm{P}_{\mathrm{rot}}=0.51 \mathrm{~d}, \mathrm{v}_{e} \sin i=90 \mathrm{~km} \mathrm{~s}^{-1}$ ) is an active, rapidly rotating single star that has recently arrived onto the main sequence. It is an ideal candidate for spectroscopic mapping techniques such as Doppler imaging as its 12.4 hour rotation period means that a large fraction of its surface can be tracked in one night, and its rotationally broadened spectral line profiles enable detailed surface maps to be reconstructed $\left(\sim 3^{\circ}\right.$ longitude resolution at the equator). Spot maps of AB Dor's surface typically show a dark spot straddling its pole (polar cap) with smaller spots co-existing at lower latitudes (e.g. Donati et al. 1999). AB Dor is also bright in X-ray wavelengths, $L_{x} / L b o l ~ 10^{-} 3$, and shows strong signs of coronal variability. ROSAT X-ray light curves of AB Dor indicate rotational modulation at the 5-13\% level (Kuerster et al. 1997).

\section{Coronal field modelling}

A time-series of high-resolution circularly polarised spectra were acquired using the AAT/UCLES/Semel polarimeter setup between 2002 Dec 11-14. These spectra are used to generate an X-ray model of $\mathrm{AB}$ Dor and to predict its quiescent $\mathrm{X}$-ray lightcurve at this epoch using the following steps (also see Fig. 1).

First, circularly polarised spectra are inverted using Zeeman Doppler imaging (Semel 1989; Donati et al 2000). The resulting magnetic field maps are extrapolated assuming a potential field distribution and that the field is open beyond $3.4 R_{*}$ (Jardine et al. 2001). If the closed corona is isothermal and in hydrostatic equilibrium the 3-D X-ray emission model of AB Dor is as depicted in Fig. 1. X-ray "snapshots" like these are used to predict the quiescent X-ray light curve of AB Dor between 2002 Dec 11-14. This model light curve predicts $\mathrm{X}$-ray rotational modulation at the $5 \%$ level in $\mathrm{AB}$ Dor's quiescent corona.

\section{Chandra/LETG spectra}

We observed AB Dor using the Chandra/LETG instrument on 2002 December 11 over $88 \mathrm{ksec}\left(1.98 \mathrm{P}_{\text {rot }}\right)$. The LETG spectra span a wavelength range from $6<\lambda<174 \AA$, with spectral resolution ranging from $460<R<2000(\mathrm{R}=2000$ at the longest wavelengths). Measuring line centroids as precisely as possible is crucial for this analysis, so it would make sense to use the best spectrally resolved lines in this dataset. In this regard, however, LETG spectra present two challenges. Firstly, the highest wavelength lines in the dataset are also the noisiest with the strongest background (Fig. 2b). This clearly affects our centroiding capability. The second challenge is that the LETG wavelengths are poorly calibrated. We compensate for these deficiencies in the following ways:

We measure the discrepancy, $\Delta \lambda$, between the observed wavelength position of the strongest emission lines and and the theoretical wavelength, $\lambda_{0}$ (using the ATOMDB database). This $\Delta \lambda$ is used to correct the wavelength position of the observed line profile and $\lambda_{0}$ is used to convert the wavelength scale into velocity- 

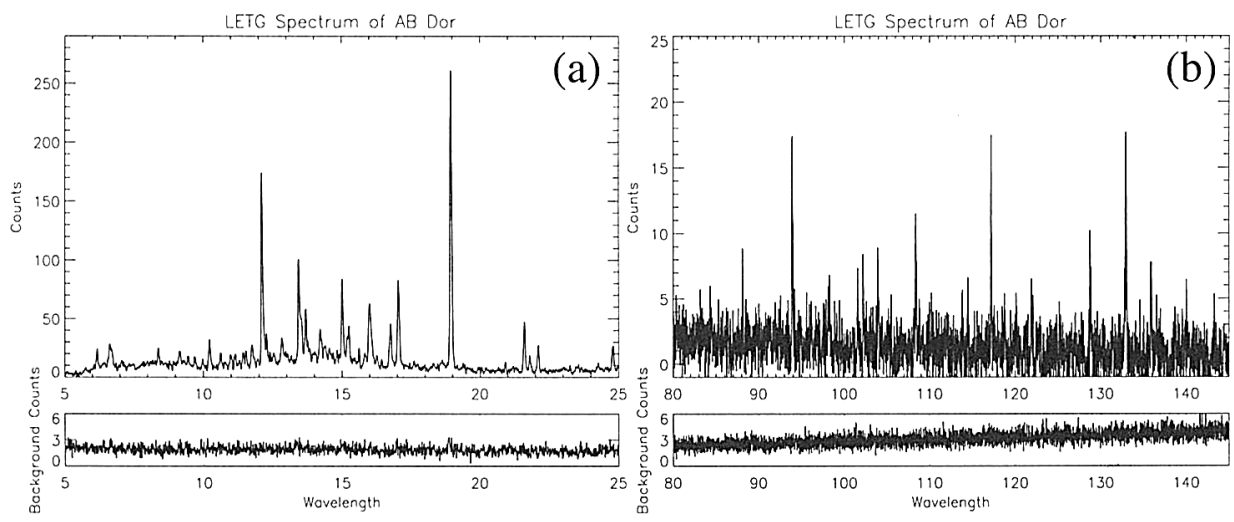

Figure 2. Chandra/LETG spectrum integrated over 88ks. Figs. a \& b show the low and high spectral resolution wavelength regions respectively along with their respective background levels.

space. The $88 \mathrm{ksec}$ exposure is then divided up into eight quarter-phase bins. To improve our centroiding capability, we integrate over the six strongest lines in the high spectral resolution region $(>88 \AA)$. This integration is done by converting the six strongest lines between $88<\lambda<133 \AA$ into velocity-space, rebinning them onto the same velocity scale and then integrating the counts from all the lines (Brickhouse, this volume). We measure line centroids by fitting Gaussians to the "summed" line at each of the eight quarter-phase bins. The centroid of this summed line profile is measured with a precision of $\pm 13 \mathrm{~km} \mathrm{~s}^{-1}$ (Fig.3). In practise, we find that the strong OvIII $18.97 \AA \AA$ line can be measured with an equivalent level of precision $\left( \pm 18 \mathrm{~km} \mathrm{~s}^{-1}\right)$ despite its relatively poor resolution.

\section{Discussion}

Fig. 3 shows the $\mathrm{X}$-ray light curve for $\mathrm{AB}$ Dor. We find that the light curve varies by $30 \%$. Initial periodogram analyses indicate that a fraction of this is indeed due to rotational modulation. We will filter this X-ray light curve in order to distinguish the rotationally modulated component from intrinsic variability. This rotationally modulated component of the light curve will be compared with the predicted light curve in Fig. 1. Our 3-D coronal model of AB Dor can also be refined, we can compensate for missing magnetic field information (e.g. in the polar cap). However, only a unipolar spot should significantly modify the coronal field distribution (McIvor et al. 2003). We will also evaluate the effect on the predicted X-ray lightcurve if the global magnetic field is non-potential using the technique described by Hussain et al. (2002).

Measurements of the centroids of the best resolved spectral lines (the summed profile derived using the above method and OviII $18.97 \AA$ line) show evidence of rotational modulation that repeats from one rotation cycle to the next. Clearly, a velocity shift with a max. amplitude of $40 \pm 13 \mathrm{~km} \mathrm{~s}^{-1}$ is not conclusive. We will add more lines from the longer wavelengths $(>88 \AA)$ to re- 


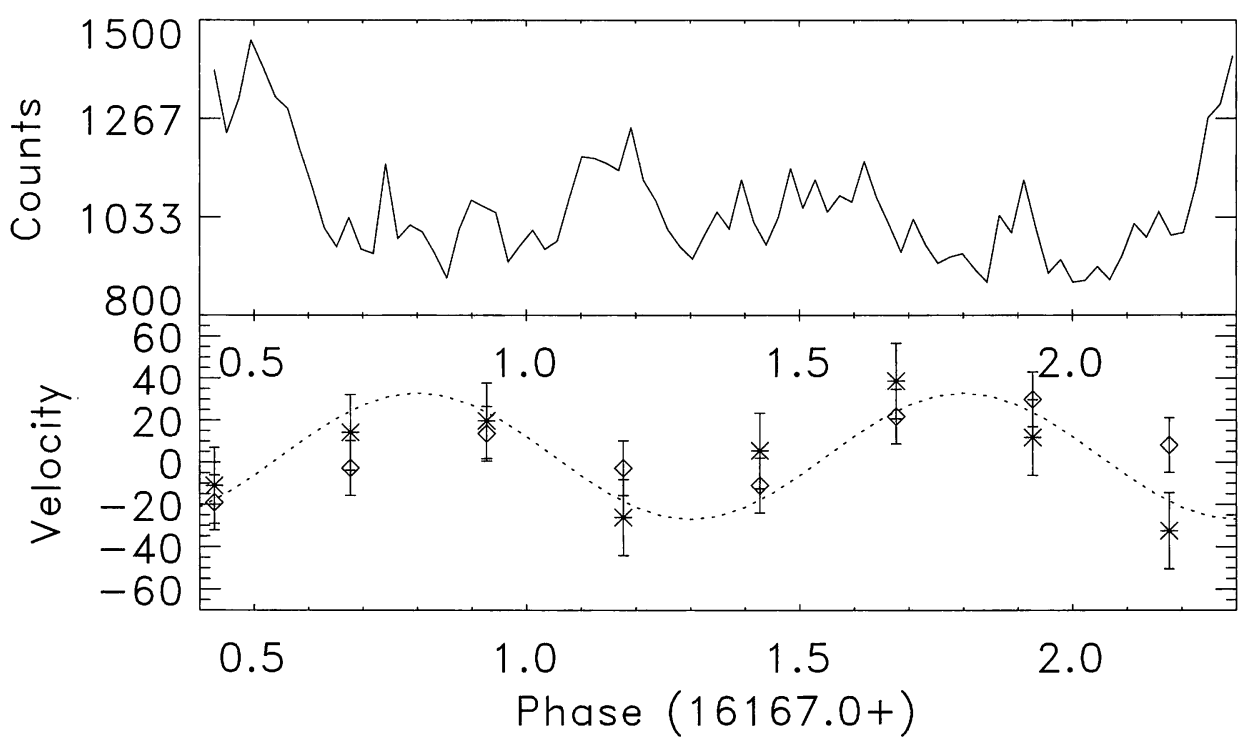

Figure 3. [top panel:] The X-ray light curve varies by 30\%. [lower panel:] Velocity shifts in line centroids of the summed line [o] and the $18.97 \AA$ line $\left[{ }^{*}\right]$ indicate rotational modulation.

duce the error bars on this measurement. The fact that both the OVIII $18 \AA$ and the summed line profile show the same trend is encouraging. This pattern of rotational modulation would indicate that the bulk of quiescent X-ray emission in AB Dor (at this epoch) originated in localised regions, most likely at high latitudes $\left(\sim 60^{\circ}\right)$. Emission originating at the stellar pole would show no rotational modulation.

\section{References}

Brickhouse, N.S., Dupree, A.K. \& Young, P.R. 2001, ApJ 562, L75

Collier Cameron, A., Jardine, M.M. \& Donati, J.-F. 2002, in ASP Conf. Proc. 277, eds. F.Favata \& J.J.Drake (San Francisco: ASP) 397

Donati, J.-F., et al. 1999, MNRAS, 302, 437

Drake, J.J., et al. 2000, ApJ, 545, 1074

Hussain, G.A.J. et al. 2002, ApJ, 575, 1078

Kürster, M. et al. 1997, A\&A, 320, 831

Jardine, M. et al. 2001, MNRAS, 324, 201

Maggio, A., et al. 2000, A\&A, 356, 627

McIvor, T. et al. 2003, MNRAS, 345, 601

Sanz-Forcada, J., Brickhouse, N.S., \& Dupree, A.K. 2003, ApJS, 145, 147

Schmitt, J.H.M.M. \& Favata, F. 1999, Nature 401, 44

Schmitt, J.H.M.M., Ness, J.-U. \& Franco, G. 2003, A\&A

Semel, M. 1989, A\&A, 225, 456 